\title{
Minquartia guianensis Aubl.: USO, ECOLOGÍA Y MANEJO EN FORESTERÍA Y AGROFORESTERÍA
}

\section{Gustav Nebel ${ }^{1}$}

\section{RESUMEN}

La especie Minquartia guianensis Aubl se encuentra diseminada por todo el neotrópico bajo, donde crece en una variedad de hábitat. En las zonas inundables de la Amazonía peruana es un producto de mucha extracción y de gran importancia socioeconómica, especialmente porque este es muy apreciado para la construcción de casas. En bosques naturales, la especie hermafrodita floreció y dio fruto casi anualmente, y se observó la producción de abundante fruto en experimentos agroforestales de 6 años en adelante. Su regeneración en bosques naturales ocurrió principalmente bajo sombra. Las plantaciones en fajas agroforestales también mostraron un mejor establecimiento de la plántula y buen crecimiento bajo sombra parcial. Los árboles adultos arriba de $10 \mathrm{~cm}$ DAP mostraron baja densidad en bosques naturales inundables (casi de 1 - 4 árboles/ha). Un modelo de relación altura - DAP mostró alturas modestas de casi $29 \mathrm{~m}$. Según un modelo de crecimiento de diámetro, de acuerdo a los datos de crecimientos de bosques naturales inundables, el crecimiento anual actual fue el más alto, de casi $25 \mathrm{~cm}$ DAP con $3,9 \mathrm{~mm} / \mathrm{año}$, mientras que el promedio de incremento anual culminó en $50 \mathrm{~cm}$ DAP con $2,7 \mathrm{~mm} /$ año. El punto ideal de volumen de producción que se alcanzó en 300 años fue de $70 \mathrm{~cm}$ DAP. En las fajas agroforestales donde la especie creció a plena luz, en tierra firme y en zonas inundables, se necesitaron casi 11 años para que se alcanzaran las alturas dominantes de $7 \mathrm{~m}$ y DAP de $8-9 \mathrm{~cm}$. Sin embargo, se observó que durante un período largo de sequía algunas plantas de buen tamaño se secaban, sugiriendo que pueden ser susceptibles a la sequía y que deberían incluirse en sistemas agroforestales donde se trabaja con sombra parcial. La reducción de las poblaciones naturales de Minquartia guianensis Aubl. y el riesgo de empobrecimiento genético debido a la extracción excesiva, exige el establecimiento de sistemas de manejo adaptados y orientados hacia los bosques naturales y a las plantaciones agroforestales. En los

1 Royal Veterinary and Agricultural University. Departament of Economics and Natural Resources Unit of Forestry, Rolighedsvej 23,1958 Frederiksberg V., Denmark Phone: +45 352822 32, Fax: +45 352826 71, E-mail: gne@kvl.dk 
bosques naturales, los métodos de silvicultura pueden ya sea aumentar la densidad de la planta o mejorar los índices de crecimiento. De cualquier manera, la gran demanda de madera de Minquartia guianensis nos sugiere que también es necesario establecer la especie en fajas agroforestales o en plantaciones.

Palabras claves: Perú, Amazonía, tierra pantanosa, tierra firme, autoecología, reproducción, dispersión, crecimiento, silvicultura.

\begin{abstract}
Minquartia guianensis Aubl. Is widespread throughout the lowland neotropics where it grows in a range of habitats. It was found to be widely extracted and socio- economically important in peruvian flood plains, as especially its durable wood is appreciated in house construction. In natural forests the hermaphroditic species flowered and fruited almost annually, and abundant fruiting was observed in agroforestry trials from 6 years age onwards. Its regeneration ocurred mainly under shade in natural forest conditions, and agroforestry and strip planting trails also showed best seedling establishment and growth under partial shade. Mature individuals above $10 \mathrm{~cm}$ DHB had low densities in natural flood plain forests (around 1 - 4 individuals/ha). A model for height DBH relationship showed development to modest heights of around $29 \mathrm{~m}$. According to a diameter growth model fitted to growth data from natural flood plain forest the current annual increment was highest around $25 \mathrm{~cm}$ DBH with $3,9 \mathrm{~mm} /$ years while the average annual increment culminated at $50 \mathrm{~cm}$ DBH with $2,7 \mathrm{~mm} /$ years. The point for optimal volume production was at $70 \mathrm{~cm}$ $\mathrm{DBH}$, which was reached in 300 years. In around 11 years dominant total heights of $7 \mathrm{~m}$ and DBH's of $8-9 \mathrm{~cm}$ were reacheding agroforestry trails where the species was grown in full light on terra firme and in flood plains. However, drying out of relatively large sized individuals was observed in some plants in connection to a prolonged dry period, suggesting that it may be susceptible to drought stress and need to be included in agroforestry systems where it is exposed to partial shade. The depletion of natural populations of M. guianensis and the risk of genetic impoverishment due to over-extraction call for adapted management systems aimed at natural forests and agroforestry/plantations. In natural forests silvicutural measures may either increase the plant density or improve the growth rates. Anyway, the high demand for M. guianensis wood suggests that it is also necessary to establish the species in agroforestry or plantations.
\end{abstract}




\section{INTRODUCCIÓN}

La especie Minquartia guianensis Albl., es un árbol que se encuentra diseminado por todos los neotrópicos bajos donde crece en una variedad de hábitat.

La madera se extrae para subsistencia y fines comerciales, especialmente para pilotes y postes de construcción, ya que está madera tiene una durabilidad excepcional. La presión extractiva es muy fuerte y, en muchos lugares, se han reducido las poblaciones naturales (Sleumer, 1984; Hunter, 1991). En consecuencia, se debe considerar opciones de manejo poniendo énfasis en la necesidad de contar con información sobre los usos, la ecología y el crecimiento de la especie.

El presente documento describe el uso y la extracción de la Minquartia guianensis, de poblaciones naturales de bosques inundables del Perú. Se describe su estructura poblacional, su crecimiento y reproducción en el entorno de tierras inundables.

Se desarrollan modelos de crecimiento en diámetro y altura. También se describen experiencias de establecimiento y crecimiento de la especie, mediante sistemas agroforestales en tierras inundables y en tierra firme.

Finalmente, se discuten los alcances y posibilidades de manejo en forestería y agroforestería.

\section{MATERIALES Y MÉTODOS}

El estudio de Minquartia guianensis se basa en los datos de una muestra de parcela permanente, en bosques inundables, y de experimentos agroforestales localizados en los alrededores de Jenaro Herrera $\left(4^{\circ} 55^{\prime} \mathrm{S}, 73^{\circ} 44^{\prime} \mathrm{O}\right)$. Este pueblo se sitúa en la parte baja del río Ucayali, en la selva baja de la Amazonía peruana, en el límite entre tierra firme al este y la zona inundada que constituye la extensa Reserva Nacional Pacaya y Samiria hacia el oeste. Además, se incluyen datos sobre la extracción de la especie en los diferentes comunidades asentadas en los límites de la Reserva Nacional Pacaya y Samiria.

Los aspectos generales de los bosques inundables del Perú han sido descritos por Kvist \& Nebel (en prensa), mientras que los detalles específicos sobre el sitio de estudio del bosque inundable fueron proporcionados por Nebel et al. (en prensa). Las fajas agroforestales son descritas por Claussi et al., (1990) y Herminio Inga (informe sin publicar). 


\section{Recolección de datos de campo}

I. Todos los árboles, con diámetro a la altura del pecho (DAP) igual a $10 \mathrm{~cm}$ o por encima de $10 \mathrm{~cm}$ (dosel superior), fueron registrados en nueve parcelas permanentes de control de una hectárea, mientras que los árboles de $1,5 \mathrm{~m}$ de altura hasta $10 \mathrm{~cm}$ DAP (dosel inferior) fueron registrados en sub-parcelas cubriendo un área de 0,64 ha.

Las parcelas de 9 hectáreas fueron igualmente distribuidas en tres tipos de bosques inundables: restinga alta, restinga baja y tahuampa. Solamente en los bosques de restinga se establecieron sub-parcelas de dosel inferior. Después del establecimiento de la parcela, a fines de 1993 se realizaron dos talas de árboles no comerciales. En cada tipo de bosque, una parcela se mantuvo intacta mientras que en los dos restantes se cortaron árboles de forma moderada en una y de forma intensa en la otra. Todos los árboles se volvieron a medir en 1994, 1995 y 1997.

Los árboles maderables también se midieron otra vez en 1998. Los registros incluyeron medidas de DAP, coordenadas y estimado en altura total.

Los datos sobre los árboles de Minquartia guianensis se utilizan para describir su comportamiento en los diferentes tipos de bosques, y para desarrollar modelos de relación altura - DAP (29 árboles) y crecimiento de diámetro (55 registros de crecimiento).

II. A fines de 1996 y comienzos de 1997, se establecieron otras parcelas permanentes en restinga alta de bosque llano inundable, formados por fajas de $40 \mathrm{~m}$ de ancho x 2,4 km de longitud, cubriendo así un área de 9,6 ha. Se registraron todos los árboles de varias especies maderables por encima de 1,5 $\mathrm{m}$ de altura. Los registros comprendían medida de DAP, coordenadas, proyección de copa en dos direcciones perpendiculares, altura total, así como altura comercial. Los DAP de todos los árboles se volvieron a medir en 1997 y 1998. Además, en 1998 todos los árboles de las especies maderables seleccionadas fueron registradas, con su DAP y coordenadas, en un área distante a $30 \mathrm{~m}$ de los linderos de las fajas establecidas anteriormente. La altura y el DAP de 109 árboles fueron utilizados para el desarrollo de un modelo de relación altura - DAP, y se usaron 190 registros de crecimiento para elaborar el modelo de crecimiento de diámetro. Entre marzo de 1997 y diciembre de 1998, se realizó al interior de las fajas una evaluación mensual de 20 árboles seleccionados de Minquartia 
guianensis, los cuales representaban a la variedad total de diámetros. Estos datos fueron empleados para describir los patrones de floración y fructificación.

III. El uso de la especie Minquartia guianensis fue evaluado en 1997, a través de una encuesta a 109 familias de 12 caseríos ubicados en 3 áreas distintas de estudio y geográficamente diferentes.

Todas las familias estaban asentadas en áreas inundables. Las preguntas se focalizaron en las especies de plantas que las familias utilizan para construcción y otros fines. Además, en una de las áreas de estudio los valores de uso - valor se calcularon para todas las especies, en base a los datos que entregaron los informantes locales respecto al potencial y uso real de los árboles de bosques inundables (Kvist et al., 1995). La extracción real de productos de los bosques inundables fue evaluada en base a un año de estudio, en las tres áreas de estudio mencionadas anteriormente. Se entrevistaron a un total de 38 familias, una vez cada dos semanas, respecto a sus actividades de extracción de las zonas inundables (Kvist et al., en este documento).

IV. En 1987, se establecieron en zonas inundables y en tierra firme fajas agroforestales que incluían a la especie Minquartia guianensis. El área inundable era una restinga alta, que dentro de un promedio anual sólo permanecía inundada poco menos que un mes. Había sido una purma durante casi 10 años previos al establecimiento del Minquartia guianensis.

Se aplicó la práctica de taungya y se plantaron, a 2 × $2 \mathrm{~m}$, plantas de raíces desnudas de aproximadamente $45 \mathrm{~cm}$ de altura. Los primeros años después de la plantación se cultivaron maíz, yuca y plátano.

El DAP, la altura y la proyección de copa fueron registrados a intervalos apropiados (Claussi et al., 1990; Centro de Investigaciones Jenaro Herrera, datos sin publicar).

En tierra firme se estableció un jardín botánico, de múltiples vástagos de árboles, en una purma en la que predominaba una vegetación arbustiva, donde anteriormente se había utilizado para el cultivo de arroz y yuca durante algunos años. Los componentes perennes lo constituían Minquartia guianensis, el árbol frutal Poraqueiba paraensis Ducke (Icacinaceae), la palmera de durazno Bactris gasipaes HBK (Arecaceae), y plátano. Los 
perennes fueron plantados como plantas de raíces desnudas a 5 x $5 \mathrm{~m}$. Las plantas de Minquartia guianensis brotaron en cada segunda fila alternando con B. gasipaes, correspondiente a un espaciamiento de $10 \times 10 \mathrm{~m}$ para cada especie. La yuca se cultivó durante los tres primeros años. Después de este periodo se estableció una cobertura de la especie Desmodium spp. que fija nitrógeno al suelo. Se realizaron evaluaciones de todos los componentes del sistema a intervalos adecuados, incluyendo medición del DAP y altura de Minquartia guianensis (Herminio Inga, dato sin publicar).

\section{Distribución, morfología y propiedades de la madera}

La especie Minquartia guianensis pertenece a la familia Olacaceae y se encuentra diseminada en la mayor parte de los neotrópicos bajos. Se encuentra presente desde Nicaragua a Costa Rica y Panamá, en las Guayanas, en la Amazonía colombiana, venezolana, ecuatoriana, peruana, boliviana y brasileña (Sleumer, 1984). Los estudios indican que crece en suelos arenosos así como en suelos arcillosos en tierra firme, en zona inundable y en bosques densos (Sleumer, 1984; Hunter, 1991).

Según Sleumer (1984), se trata de un árbol entre pequeño y grande que crece recto hasta alcanzar alturas moderadas de 10-20 m. Hunter (1991) reportó alturas de hasta casi $30 \mathrm{~m}$, de acuerdo a sus observaciones realizadas en lugares de bosques naturales de tierra firme e inundable de Jenaro Herrera, en la Amazonía peruana. Clark \& Clark (1992), también caracterizaron a la especie como un árbol de dosel, que probablemente alcance un diámetro de hasta $1 \mathrm{~m}$ o más, aunque ahora es muy difícil encontrar árboles grandes debido a la excesiva extracción.

Los troncos grandes se caracterizan por estar a veces perforados y por presentar ramas muy profundas. Minquartia guianensis se reconoce fácilmente por su tronco de corteza marrón grisácea, de escamas rectangulares y ramas rectas verticales, y sus hojas tan distintas.

La especie es hermafrodita, con frutas drupa elipsoide de aproximadamente 2,5 x 1,5 cm. Aróstegui \& Díaz (1992) calcularon el peso de 1000 semillas frescas en $1,89 \mathrm{~kg}$, Sleumer (1984) hizo una detallada descripción taxonómica de esta especie. 
La madera de Minquartia guianensis es excepcionalmente durable, se sabe que ha durado de 30 a 40 años en la tierra sin mostrar ninguna señal de debilitamiento, y es por eso que se le prefiere mucho para su uso como postes y pilotes en la construcción (Sleumer, 1984; Hunter, 1991). Es muy resistente a los organismos destructivos de madera, probablemente debido a que contiene substancias fungitóxicas, y también resistente a los ataques de las termitas, lo cual se atribuye a su elevada densidad de madera y probable contenido de repelente o extractos tóxicos (Scheffer \& Duncan, 1947; Bultman \& Southwell, 1976).

Minquartia guianensis tiene varios nombres vernaculares: en Brasil: acapú, acari, arariuba, acariquara, a roxa; en Costa Rica: manú negro; en Guayana francesa: maka, minquar, paicoussa rouge; en Guyana: man wood, mincoa; Panamá: man wood, nispero negro; en Perú: huacapú; en Surinam: alataudu, alata-weri, wananin; y en Venezuela: urana-u-yek (Sleumer, 1984).

\section{RESULTADOS}

\section{Uso local}

Minquartia guianensis se usa de manera predominante para fines de subsistencia, como postes para la construcción de casas y como medicina contra la malaria, para combatir a los parásitos intestinales y contra los resfriados. Estos horcones también se expenden en los mercados locales de los pueblos más grandes. Según los resultados del censo III, la especie está cerca de una calificación óptima de uso-valor, para usos como postes (horcones) y vigas, y se indica que cubre más del 50\% de la demanda de postes entre los encuestados.

También se encontró que $M$. quianaensis, como poste, predominaba entre las familias encuestadas en las zonas de estudio 1 y 2 durante el censo III (Tabla 1). En la zona de estudio 3 se encontró que la proporción de postes hechos de $M$. guianensis era baja, y que éstos eran mayoritariamente viejos (de 30-40 años). Esto nos indica que la especie ha disminuido, lo cual concordaba con la información brindada por la gente mayor, quienes decían que hubo un tiempo en que eran abundantes las poblaciones naturales. 
En las zonas de estudio 1 y 2 , las familias entrevistadas en el censo III, sobre sus actividades extractivas de las zonas inundables, indicaban que el $62-84 \%$ de los postes (horcones) fueron extraídos de los árboles Minquartia guianensis. En la zona de estudio 3 ningún poste fue hecho de esta especie, sugiriendo nuevamente que la especie había disminuido. En la zona de estudio 2 aparece que la especie ha sufrido un proceso de sobre-explotación, ya que la proporción de postes extraídos es menor que la proporción observada en las casas (Tabla 1).

\section{Reproducción}

La fenología de Minquartia guianensis, observada en un ambiente de bosque natural durante el censo III, mostró un patrón monomodal de floración y fructificación durante un periodo de 4 meses, de setiembre a diciembre de 1997 (Figura 1). En 1988, los árboles se mostraron infértiles, lo que quizá se debió a la larga sequía. En 1997, la floración y la fructificación duraron 2 meses, y se registraron en casi el 70\% de los árboles que tenían un DAP mayor que el del árbol fértil más pequeño. El árbol más pequeño con flor o fruto, tenía un DAP de $22,8 \mathrm{~cm}$ a $28,7 \mathrm{~cm}$ respectivamente. La floración y la fructificación de 1997 coincidieron con el período menos lluvioso y más caluroso del año, y ocurrió fuera del período de inundación de las zonas bajas, y duró desde diciembre hasta abril.

Las plantas que crecieron en fajas agroforestales, florecieron y fructificaron aproximadamente a partir de los 6 años. Los períodos fértiles en las fajas inundables y en las de tierra firme, coincidieron más o menos con el patrón observado en los bosques naturales inundables.

No obstante, en 1998, también se observó que había floración y fructificación en las fajas agroforestales donde antes eran infértiles las poblaciones de bosques naturales inundables. Esto podría deberse a la existencia de condiciones más favorables para la producción de frutas cuando los árboles crecen en un ambiente de plena luz. La producción de fruta fue considerable, ya que muchos de los árboles de agroforestería produjeron cientos de semillas.

Las semillas de Minquartia guianensis probablemente son dispersadas por pequeños mamíferos y aves grandes, como los tucanes, a los cuales les encanta el dulce pericarpio. Sin embargo, la mayoría de las semillas caen 
debajo de las copas de los árboles madre. En el bosque natural, las plántulas y retoños solamente brotaron bajo condiciones de sombra, pero también pueden afirmarse y crecer a plena luz. Sin embargo, en las fajas agroforestales se observó un mejor establecimiento y crecimiento de las plántulas, cuando se mezclan o en los linderos del campo sobre el cual la vegetación circundante proyecta una sombra parcial. Esto nos sugiere que las etapas juveniles de Minquartia guianensis son más exitosas cuando hay sombra. Un experimento que consistía en exponer las plántulas crecidas en viveros a los diferentes períodos de inundaciones, mostró que todas las plántulas morían cuando eran expuestas durante un mes y medio a continuas inundaciones, mientras que sobrevivió el 20\% de aquéllas que sólo fueron expuestas durante 1 mes bajo estas condiciones.

\section{Crecimiento y estructura de la población}

Minquartia guianensis registró densidades bajas entre los árboles de más de $10 \mathrm{~cm}$ de DAP, en tres bosques naturales inundables que se inventariaron en el censo I (Tabla 2). Sin embargo, su densidad podría ser mayor en condiciones normales, ya que el lugar del estudio bien pudo haber estado sometido a actividades extractivas. En bosques naturales e inundables adultos, la distribución del diámetro de los árboles mostró un patrón "J" invertido, indicando que se regeneró bajo sombra (ver Figura 2). En la Tabla 2 también se indica que fue insignificante el crecimiento durante el período de cuatro años de estudios.

Un modelo de relación altura - DAP de Minquartia guianensis, se adecuó a los datos de campo (recogidos en los censos I y II), mediante una ecuación monomolecular "Mitcherlich" que utiliza un método no lineal. Se observó un precario desarrollo de altura (Figura 2).

$$
h=28,45 .\left(1-0,9198 \cdot e^{(-0,03725 . d)}\right)
$$

Donde h es la altura (m) y d es DAP (cm).

El crecimiento del diámetro de Minquartia guianensis, en clases de diámetro, se calculó en base a los registros realizados en las parcelas permanentes (Censo I y II), de los cuales, la clase de $30 \mathrm{~cm}$ DAP mostró el promedio más alto de incremento de diámetro (Tabla 3). El tiempo que los árboles necesitan para alcanzar $70 \mathrm{~cm}$ DAP es de 234 años y 57 años 
según los índices de crecimiento DAP intermedio y máximo de las clases de diámetro.

Los registros de la parcela permanente (Censo I y II) se utilizaron para definir un modelo de crecimiento de diámetro mediante el uso de la ecuación de Wykoff (Vanclay, 1994) teniendo el DAP como la única variable:

$$
\Delta \mathbf{d}=\sqrt{\mathbf{e}^{\left(-6,094+1,49 \cdot \ln (\mathrm{d})-0,00095341 . \mathrm{d}^{2}\right)}}
$$

donde $\Delta \mathrm{d}$ es el incremento de DAP en cm por año, y d es el DAP en $\mathrm{cm}$.

Unas pruebas $\mathrm{F}$ estandarizadas, mostraron una relativa adecuación buena del modelo $\left(\mathrm{R}^{2}=0,35, \mathrm{P}<0,0001\right)$. El modelo pronostica un punto máximo entre 25 y $50 \mathrm{~cm}$ DAP, respectivamente en los índices de crecimiento de diámetro actuales y de promedio anual (Figura 3). El crecimiento del diámetro es lento, lo que probablemente sería una característica inherente a la especie, pero también la causa podría deberse al hecho de que la mayoría de los árboles crecen bajo la sombra. Sin embargo, a pesar de incluir la posición de la copa de los árboles de acuerdo a la clasificación de Dawkins, no se logró mejorar la adecuación del modelo. Al combinarse el modelo de crecimiento de diámetro (2) con el modelo de altura - DAP (1) y el factor de forma 0,5 , que es utilizado para un número de especies de árboles tropicales (Cannell, 1984), se obtiene un crecimiento de volumen con índices de crecimiento actuales y anuales que oscilan entre 45 a $70 \mathrm{~cm}$ DAP, respectivamente (Figura 3). Esto índica un diámetro de corte para obtener un excelente volumen de producción a $70 \mathrm{~cm}$ DAP, lo cual se alcanza casi a los 300 años. Sin embargo, el lento crecimiento en diámetros menores, pronosticado por el modelo, puede subestimar el crecimiento del diámetro de los árboles adultos, ya que al momento de adecuar el modelo, se incluyeron árboles jóvenes de crecimiento lento que probablemente tenían elevados índices de mortalidad.

Al comparar los árboles de Minquartia guianensis, de senderos agroforestales y bosques naturales, se encontró que los DAP grandes se obtienen a una altura determinada, y que es mayor el crecimiento del diámetro en los árboles de fajas agroforestales (Compare Figura 2 y 3 con 
Figura 4). Esto demuestra que la especie se pueda establecer y crecer bajo condiciones de plena luz, aunque, principalmente, su regeneración en el bosque natural está confinado a condiciones de sombra. También sugiere que cuando se planta Minquartia guianensis en fajas agroforestales, se puede alcanzar los diámetros aceptables para fines de construcción en un relativo corto periodo de tiempo. Sin embargo, como se mencionó anteriormente, se observó un establecimiento superior y excelente crecimiento cuando los árboles agroforestales fueron expuestos a un poco de sombra especialmente durante los primeros años. De hecho, los árboles grandes (alrededor de $8 \mathrm{~cm}$ DAP) y aislados que crecían a plena luz, en suelos agroforestales de tierra firme, se secaron de forma repentina sin mayores señales de daños.

\section{DISCUSIÓN}

La intensa extracción de la madera de Minquartia guianensis, para subsistencia y comercio, especialmente para postes (horcones) en la construcción de casas, ha traído como consecuencia una reducción masiva de sus poblaciones naturales. Las áreas donde la especie aún se encuentra en poblaciones naturales proveen el $80 \%$ de los postes utilizados en la zona (Tabla 1). Se observaron valores menores de la extracción real versus uso anterior de la especie, en zonas de acceso limitado y permanente a los bosques naturales que contienen Minquartia guianensis (Tabla 1). Esto pone en énfasis la necesidad de proveer información sobre su ecología y crecimiento, así como de poner en consideración la organización de las actividades de extracción; en los siguientes párrafos se discuten aspectos de éstos temas. Las técnicas mejoradas para la construcción de casas, incluyendo medidas apropiadas de la madera y en lo posible el uso de maderas menos durables, probablemente, también contribuirían a aliviar la presión existente sobre la madera durable y de lento crecimiento de Minquartia guianensis.

La floración y la fructificación de Minquartia guianensis se observó durante el período seco y sin inundación del año. Esto coincide más o menos con las observaciones reportadas por Aróstegui \& Díaz (1992), sobre 12 árboles estudiados, de 1985 a 1989, en los alrededores de Jenaro Herrera. Los árboles presentaban en promedio 29 $\mathrm{cm}$ de DAP, florecieron entre junio - julio y fructificaron de julio a setiembre. La vigorosa fructificación en los bosques naturales, aunque baja en algunos años, y la temprana fertilidad de las plantaciones nos indica que la disponibilidad de semilla podría ser un problema pequeño para el manejo. También implica que los programas sencillos de mejoramiento genético, servirían como una solución rápida. 
Se encontraron plántulas y árboles jóvenes de Minquartia guianensis, de forma predominante, bajo sombra en los bosques naturales inundables, pero también crecieron a plena luz en las fajas agroforestales, aunque aparentemente se desarrollaron mejor en lugares donde las plántulas estaban expuestos a sombra parcial. Los experimentos de transplante de plántulas crecidas en viveros bajo plena luz y en fajas en línea, mostraron un desarrollo superior de la plántula bajo sombra (Aróstegui \& Díaz, 1992). Clark \& Clark (1992) estudiaron la historia de vida de Minquartia guianensis y de 5 especies de árboles emergentes en bosques naturales de tierra firme. Se descubrió que las etapas juveniles de la especie estaban asociadas a la baja iluminación de la copa y de los bosques en la fase adulta. Los índices de mortalidad eran relativamente bajos y declinantes al incrementarse el tamaño, y no se observaron diferencias en los índices de mortalidad entre las plántulas que crecían bajo diferentes condiciones de micrositio. Se confirmó la impresión que Minquartia guianensis es una especie tolerante a la sombra, mediante los estudios ecofisiológicos de plántulas conducidos por Vieira (1996). El evaluó sus reacciones alométricas, morfológicas y fisiológicas en diferentes condiciones de luz y de nutrientes, y encontró que sigue una estrategia de tolerancia flexible. La especie manifestó poca plasticidad respecto a las diferentes condiciones de luz y de nutrientes. Se observaron resultados notables en el aumento de la altura a partir de la combinación de sombra moderada con altos niveles de nitrógeno, y una baja reacción ante los cambios bruscos de los regímenes de luz.

Sin embargo, se consideró que Minquartia guianensis posee un nicho fundamental amplio, ya que los índices de crecimiento mejoraron con la radiación activa fotosintética (Vieira, 1996). La muerte de las plántulas de Minquartia guianensis que fueron expuestas a inundaciones continuas de más de un mes y medio, indica que la duración de la inundación limita su diseminación en ambientes inundables. En consecuencia, los cambios considerables en la duración y el nivel de inundación anual de las llanos inundables amazónicos (Irion et al., 1997) implica que la especie se pueda establecer en sectores que se inundan periodos más largos que el promedio, asumiendo que los árboles de gran tamaño generalmente son más resistentes a la inundación (Gill, 1970). Asimismo, el establecimiento rápido y el crecimiento inicial, inmediatamente después del final de la inundación, permitirá que los árboles jóvenes alcancen un tamaño tolerante antes del comienzo de la siguiente inundación. Estos mecanismos podrían explicar por que Minquartia guianensis se encuentra presente en los bosques llanos inundables que tienen promedios anuales de inundaciones de hasta 4 meses, a pesar que sus plántulas son susceptibles a las inundaciones.

En el contexto del manejo, las implicaciones de las características de los árboles 
jóvenes indican que el establecimiento y el temprano crecimiento de Minquartia guianensis debería ocurrir preferiblemente bajo sombra parcial o total, ya que la especie se establece bajo tales condiciones en los bosques naturales, y se demostró que su crecimiento inicial y su establecimiento son superiores bajo estas condiciones. La repentina sequedad de árboles de gran tamaño (de aproximadamente $9 \mathrm{~cm}$ DPA), que crecieron solitarios y bajo plena luz en las fajas agroforestales, también nos sugiere que se debería tener cuidado al introducir la especie bajo tales condiciones. En estos casos la mortalidad pudo haber sido causada por la combinación de sequía y la total exposición a la luz. Cuando se intente establecer la especie en lugares llanos que se inundan por mucho tiempo, hay que recordar, que los años consecutivos de poca inundación que permita a los árboles crecer hasta tamaños tolerantes a la inundación, podría ser un pre-requisito para lograr un establecimiento exitoso. El transplante de plántulas de gran tamaño, quizás podría ser una manera para superar la mortalidad inducida por la inundación.

En los bosques naturales de llanos inundables, la densidad de árboles adultos de Minquartia guianensis fue baja (Tabla 2), quizás en parte debido a la extracción. El crecimiento fue relativamente lento como se demostró con las observaciones de clases diámetro (Tabla 3). Similarmente, el modelo (2) de incremento de diámetro (Figura 3) pronosticó índices de crecimiento anuales de sólo 1 - $4 \mathrm{~mm} / \mathrm{año}$ para los árboles con 5 - $50 \mathrm{~cm}$ DAP. Clark \& Clark (1992), estudiaron a Minquartia guianensis en los bosques de tierra firme en Costa Rica, y también observaron modestos índices de crecimiento mediano y máximo de $1,4-2,8$ y 7,5 - 12,5 mm/año en clases de diámetro de 4 a $70 \mathrm{~cm}$ DAP, respectivamente. Encontraron una correlación entre el crecimiento del diámetro y las medidas de iluminación de copa y las copas sobre los árboles, que contrasta con el fracaso del presente estudio para lograr el desarrollo de un mejor modelo que incluía valores para la posición de la copa.

Si no se aplican las medidas agroforestales necesarias para potenciar la densidad y el crecimiento, sólo contaríamos con la capacidad limitada de los bosques llanos naturales inundables para proveer madera de Minquartia guianensis. Esta afirmación se puede graficar con el siguiente ejemplo: dada una densidad natural de, digamos, 2 árboles adultos por hectárea y una intención de corte del tamaño de $30 \mathrm{~cm}$ DAP (por debajo del punto ideal de volumen de producción de $70 \mathrm{~cm}$ DAP), sólo sería posible cosechar un árbol por hectárea cada 75 años según el modelo de crecimiento (2), si se tuviese que mantener una densidad de 2 árboles por hectárea. La base de datos mostró que las familias extrajeron un promedio cercano a 3 postes por año. Suponiendo que estos fueron provistos por un árbol de $40 \mathrm{~cm}$ DAP, significa que cada familia necesitaría 75 hectáreas de bosques llanos inundables para satisfacer sus necesidades de postes para la construcción de casas. 
En consecuencia, en vista de los escasos recursos forestales y de la creciente demanda de Minquartia guianensis para subsistencia y fines comerciales, es necesario el desarrollo y la aplicación de sistemas agroforestales para reforzar el establecimiento y crecimiento de la especie en los bosques naturales. Probablemente, se podría fácilmente incrementar los índices de densidad y crecimiento en base a la existencia de abundantes semillas que mayormente caen debajo y cerca de las copas de los árboles madres. Aquí podrían haber muchas plántulas que toleran la sombra, aunque parece que su crecimiento se reduce en aquellos ambientes de mucha sombra que son muy frecuentes. Por lo tanto, sería ventajoso diseminar las plantas por todo el bosque y particularmente en lugares que tengan poca sombra, es decir, debajo del dosel de especies colonizadoras. Esto se podría hacer plantando plántulas crecidas en viveros, pero quizás más fácilmente replantando plántulas sacadas de poblaciones cercanas a los árboles madres, teniendo en cuenta, que éstos crecen poco cuando están expuestas a cambios brusco del régimen de luz (Vieira, 1996).

Considerando que la extracción selectiva de madera en los bosques naturales no permite suficiente luz para garantizar niveles altos de crecimiento, sería factible cultivar para despejar alrededor de los árboles de Minquartia guianensis, aunque no es esencialmente importante que exista iluminación directa sobre la copa. Esto podría ayudar a acelerar el crecimiento del diámetro, a pesar de que la inclusión del valor de posición de la copa, en el modelo de crecimiento del presente estudio, no mejoró la calidad del mismo.

En base a los parámetros biométricos, obtenidos de las fajas agroforestales en zonas inundables y de tierra firme, se observó que Minquartia guianensis se desarrolló razonablemente bien durante los primeros 11 años del estudio (Fig. 4). Sin embargo, la repentina mortalidad de árboles de gran tamaño (de cerca de $8 \mathrm{~cm}$ DAP), crecidos bajo plena luz en las fajas de tierra firme, nos indica que pueden ocurrir problemas cuando los sistemas agroforestales no están bien diseñados. La observación del fenómeno durante una sequía inusual y prolongada, sugiere que la exposición de plantas relativamente jóvenes a la luz total, descontrola a los árboles de una manera excesiva, y empiezan a secarse hasta morir. Cabe resaltar, sin embargo, que quizás otros factores provocaron el fenómeno y que es necesario realizar más estudios. De cualquier forma, habría que experimentar con los sistemas agroforestales que combinan Minquartia guianensis con otras especies de árboles, de mayor tamaño y de crecimiento rápido que producen sombra parcial. No sabemos si seguiría creciendo tan rápido, como se observó en el presente estudio, pero sí se lograría reducir el riesgo de muerte por sequedad. 
El aumento de los índices de crecimiento podría incrementar la productividad en forestería y agroforestería y, así, se reduciría el riesgo de disminución y empobrecimiento genético de las poblaciones naturales causados por la sobre-extracción.

Sin embargo, como la madera de Minquartia guianensis es apreciada por su durabilidad, vinculada a sus componentes químicos y a su densidad, se podría asumir que la resistencia de la madera de rápido crecimiento sea inferior en vista de la baja densidad y poca concentración de componentes químicos. Por ahora, no existe información al respecto, haciéndose necesario una investigación adicional y un enfoque conservacionista para el diseño de los sistemas de manejo. Sobre este punto, es apropiado señalar las posibles técnicas de ahorro de madera en la construcción de casas tradicionales: frecuentemente se observa el uso de maderas muy grandes y en algunos casos se podría utilizar como alternativas especies menos resistentes que Minquartia guianensis, es decir, en la parte de los postes que no estén en contacto con el suelo, y todos los elementos que no estén expuestos a organismos que destruyen madera o a los ataques de las termitas. 


\section{BIBLIOGRAFÍA}

ARÓSTEGUI, A. V. , DÍAZ, M. P. 1992. Propagación de especies forestales nativas promisorias en Jenaro Herrera. Publifor, Lima, 119 pp.

BULTMAN, J. D., SOUTHWELL, C. R. 1976. Natural resistance of tropical American woods to terrestial woods - destroying organisms. Biotropica, 8 (2): $71-95$.

CANNELL, M. G. R. 1984. Woody biomaass of forest stands. Forest Ecology and Management, 8: 299 - 312.

CLARK, D. A., CLARK, D. B. 1992. Life history diversity of canopy and emergent trees in a neotropical rain forest. Ecological Monographs, 62 (3): 315 344.

CLAUSSI, A., MARMILLOD, D., BLASER, J. 1992. Descripción silvicultural de las plantaciones forestales de Jenaro Herrera. Instituto de Investigación de la Amazonía Peruana, Iquitos, 334 pp.

GILL, C. J. 1970. The flooding tolerance of woody species a review. Forestry Abstracts, 31 (4): 671 - 688.

HUNTER, J. R. 1991. Observations on the growth, ecology and uses of Minquartia guianensis, a humid tropical tree. The International Tree Crops Journal, 6: $221-238$

IRION, G. , JUNK, W. J., DE MELLO, J. A. S. N. 1997. The large central amazonian river floodplains near Manaus: geological, climatological, hydrological, and geomorphological aspects. In: W. J. Junk (Editor), The central Amazon floodplain. Ecology of a pulsing system. Springer - Verlag, Berlin, pp. 23 46.

KVIST, L. P., ANDERSEN, M. K., HESSELS 4 E, M., VANCLAY, J. 1995. Estimating use - values and relative importance of Amazonian flood plain trees and forest to local inhabitants. Commonwealth Forestry Review, 74 (4): 293 - 300.

SCHEFFER, T. C. DUNCAN, C. G. 1947. The decay resistance of certain Central American and Ecuadorian woods. Tropical Woods, 92: 1 - 24. 
SLEUMER, H. O. 1984. Flora Neotropica, Monograph Number 38, Olacaceae. The New York Botanical Garden, New York.

VANCLAY, J. K. 1994. Modelling forest growth and yield. Aplications to mixed tropical forests. CAB International, Wallingford, $312 \mathrm{pp}$.

VIEIRA, G. 1996. Gap dynamics in managed Amazonian forest: Structural and ecophysiological aspects. $\mathrm{PhD}$ dissertation. Linacre College and Oxford Forestry Institute, Departament of Plant Sciences, University of Oxford. $162 \mathrm{pp}$. 


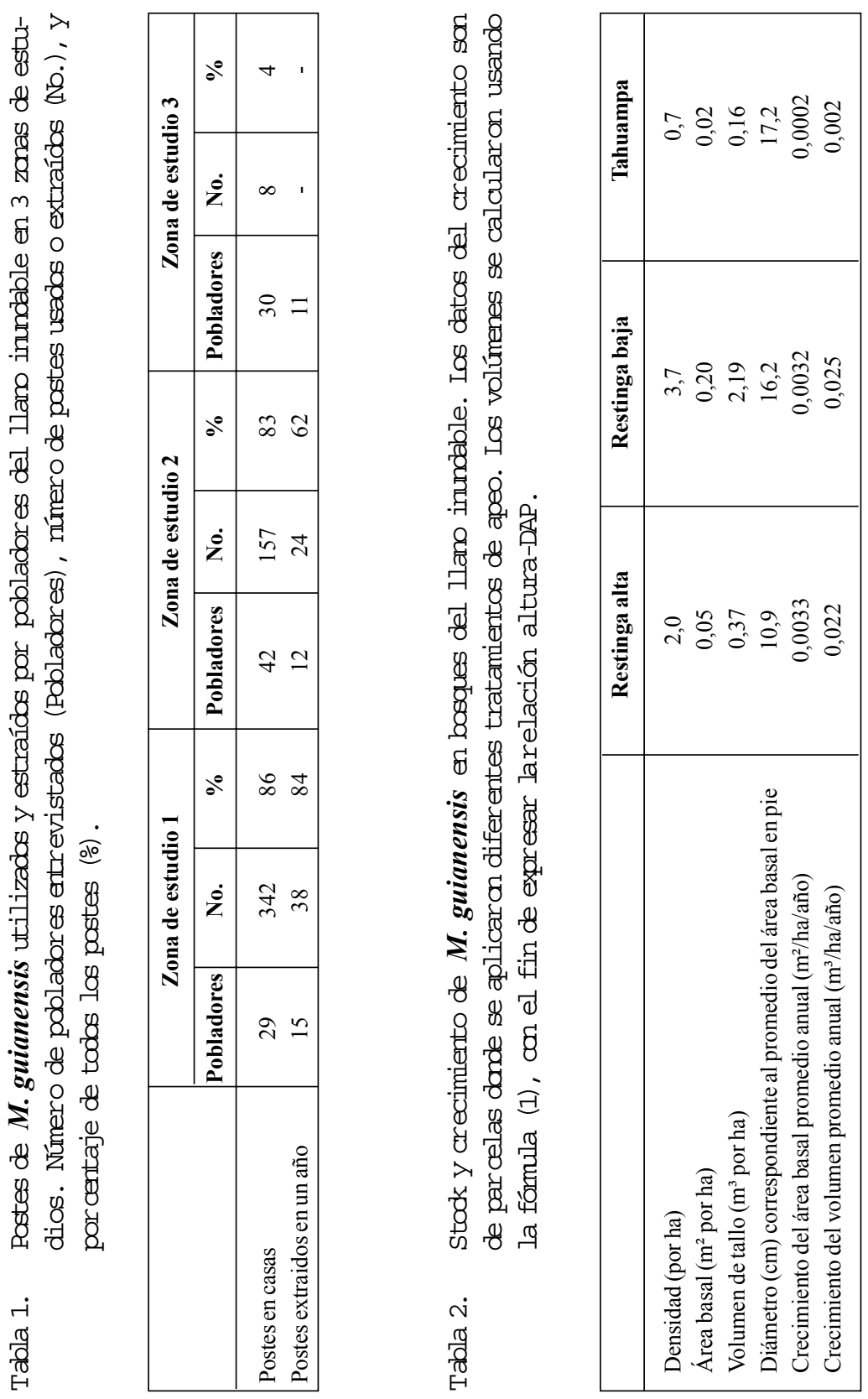


\&.

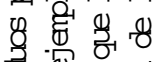
:0ृ 然 o 0 定

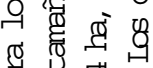
\& \& 8 . is

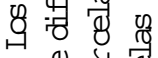
$\therefore$. 8 ह 8

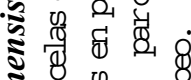

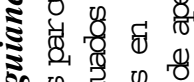

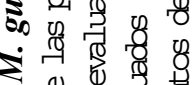
की की

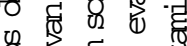

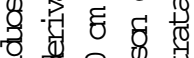
to 80 on : न

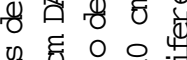

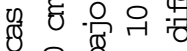
각ㅇ 용 음 要 잉 \% 의 पु वा णू प्र न्व 0 मे

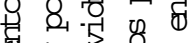
类者 है

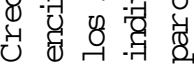

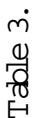

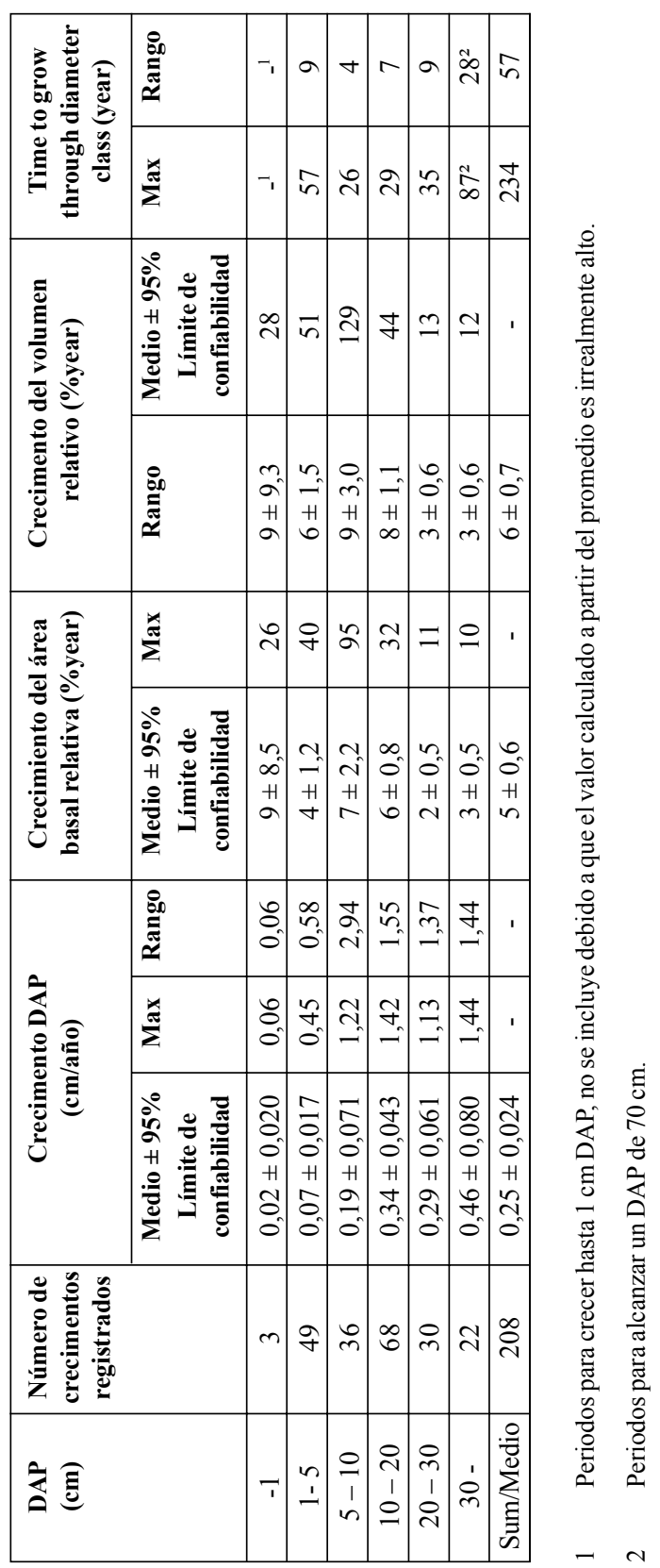




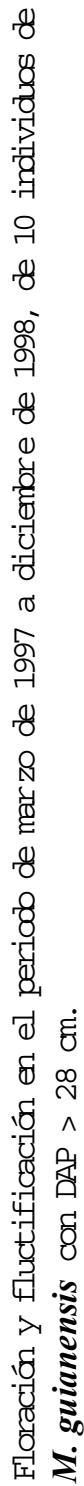

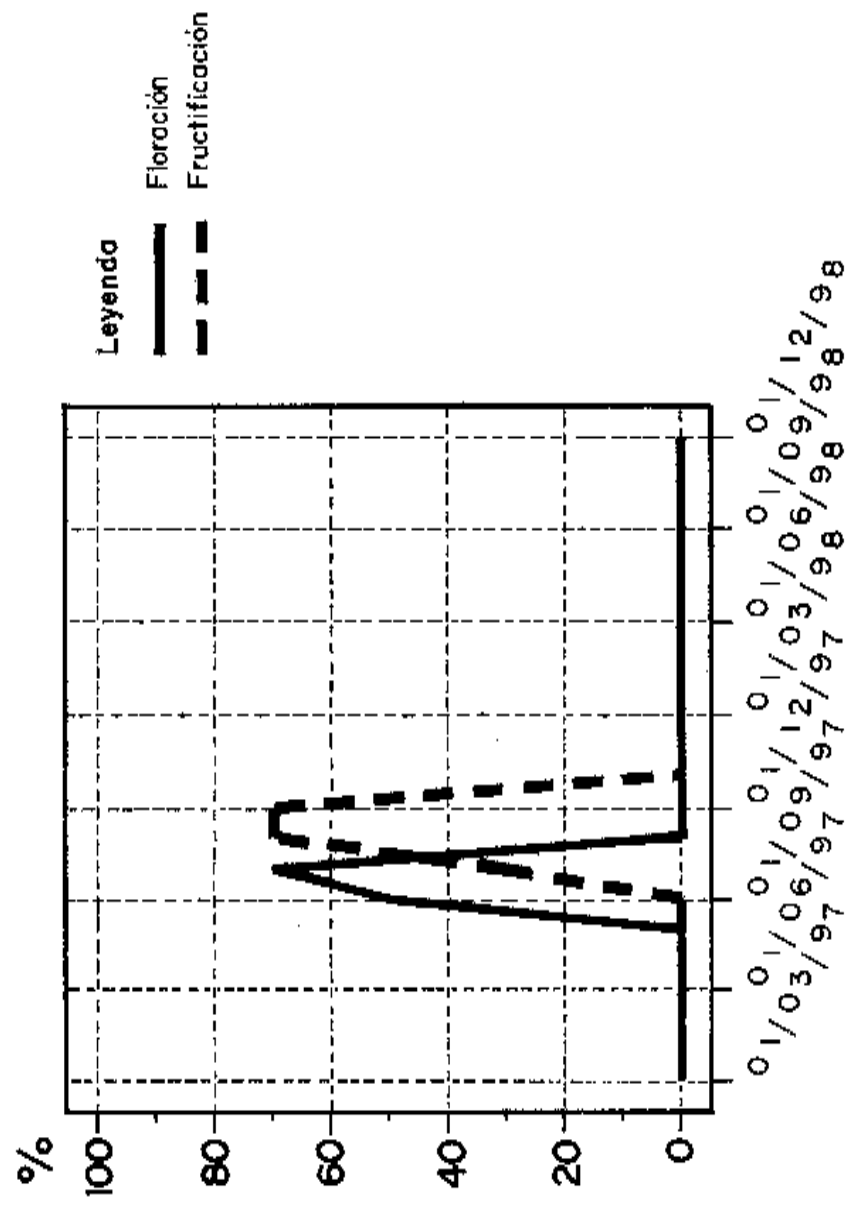

त् 


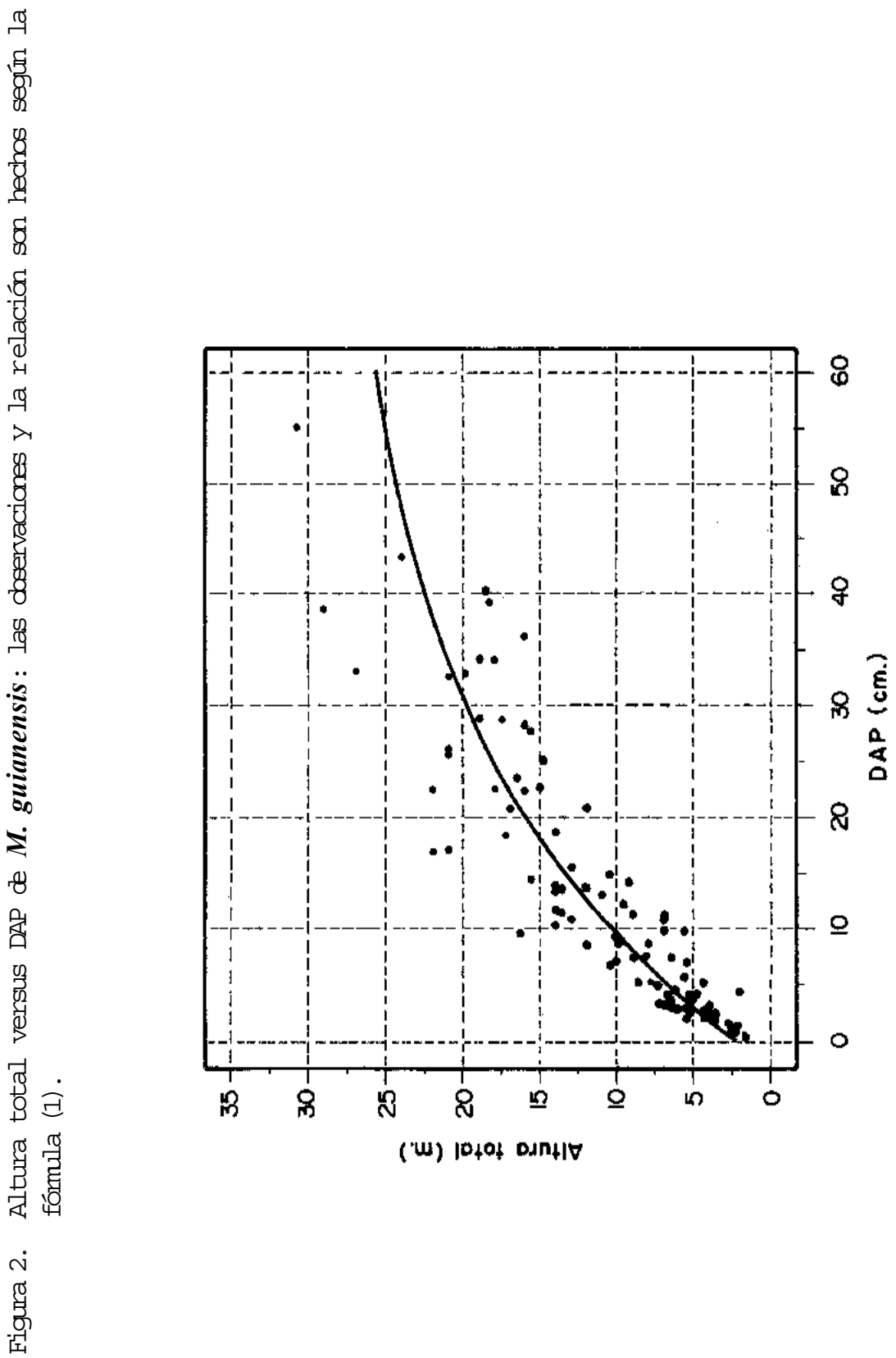



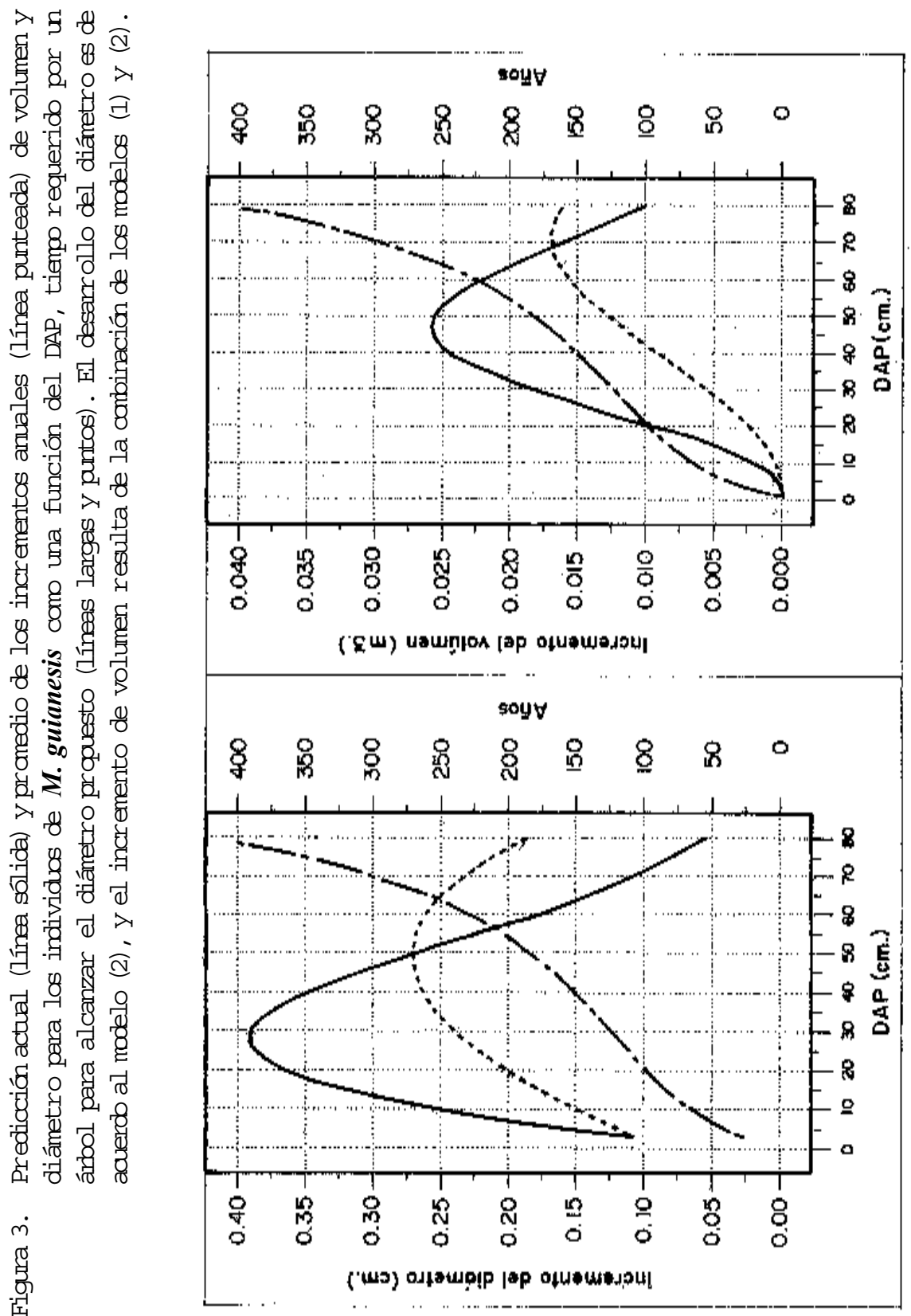


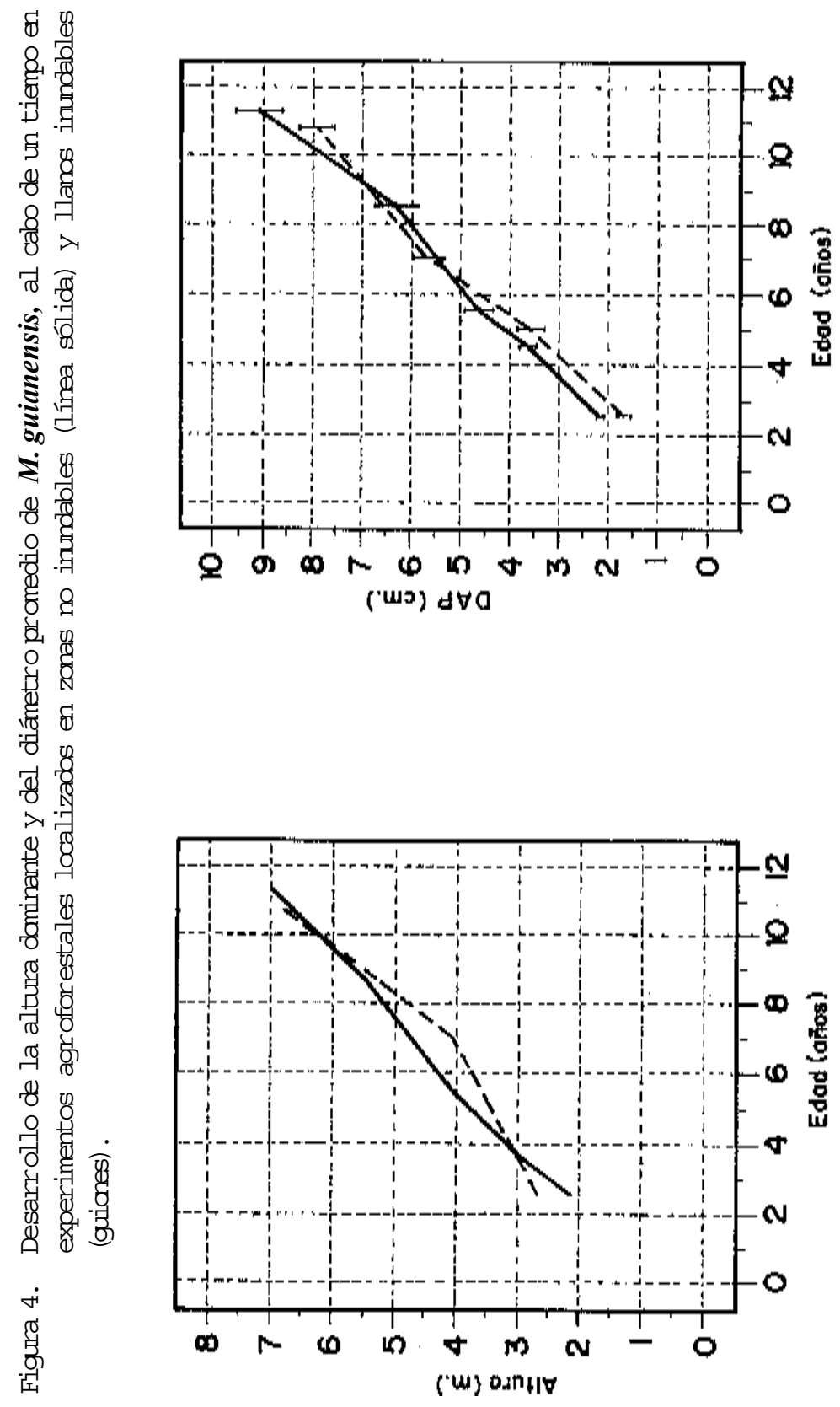

